\title{
MULTIPLICITY AND NUMBER OF PARTS IN OVERPARTITIONS
}

\author{
SYLVIE CORTEEL AND PAWEŁ HITCZENKO
}

\begin{abstract}
The purpose of this paper is to study the parts, part sizes and multiplicities in overpartitions using combinatorics, probabilities and asymptotics. We show that the probability that a randomly chosen part size of a randomly chosen overpartition of $n$ has multiplicity $m$ or $m+1$ approaches $1 /(m(m+1) \ln 2)$ and that the expected multiplicity of a randomly chosen part size of a randomly chosen overpartition of $n$ approaches $\ln n /(4 \ln 2)$ as $n \rightarrow \infty$.
\end{abstract}

AMS classification : 05A17, 60C05, 11P82.

Keywords : Partitions, Combinatorial probability.

\section{INTRODUCTION}

An overpartition of the integer $n$ is a non-increasing sequence of positive integers that sums to $n$ where the last occurrence of an integer can be overlined. The quantity $n$ is called the weight. Given an overpartition $\left(\lambda_{1}, \ldots, \lambda_{k}\right)$ we call the $\lambda_{i}$ s the parts. The number of part sizes is the number of distinct integers that occur in the partition and the multiplicity of the part $i$, denoted by $m_{i}(\lambda)$, is the number of occurrences of the part $i$ in $\lambda$ (overlined or not). For example, $\lambda=(5, \overline{4}, 2,2, \overline{2}, 1)$ is an overpartition of 16 . It has 6 parts, 4 part sizes and the multiplicity of the part 2 is 3 . We denote by $\bar{p}(n)$ the number of overpartitions of $n$.

These objects were used under different names and guises, see for example [17]. Overpartitions were named by Corteel and Lovejoy $[4,6,7]$ and used to give the first combinatorial proofs of the $q$-Gauss identity and Ramanujan's ${ }_{1} \psi_{1}$ summation. They were used by Yee [24] to give a more direct proof of Ramanujan's ${ }_{1} \psi_{1}$ summation. They were also shown to be a good tool to discover infinite families of Frobenius partitions whose generating function are infinite products [8]. They are also studied using $q$-series and number theoretical techniques [20,21, 22]. Recently they were also related to some physical problems as they are in bijection with jagged partitions $[2,11]$. Afterwards they were successfully used by Bessenrodt and Pak [3] under the name joint partitions.

The purpose of this work is to study parts in overpartitions. As is customary we consider the uniform probability measure on the set of all overpartitions of $n$, and by random overpartition of $n$ we will mean a partition picked according to that measure.

This work was supported in part by the NSF Grant INT-0230800. The work of the first author was also partially supported by the ATIP Jeune Chercheur CNRS. The work of the second author was also partially supported by the NSA Grant MSPF-02G-043. 
From the definition, some facts are obvious :

- The probability that a randomly chosen part size of a randomly chosen overpartition of $n$ is overlined is $1 / 2$.

- The expected weight of the overlined parts counted with their multiplicity of an overpartition of $n$ is $n / 2$.

Both of these facts follow from an involution on overpartitions. Given an overpartition, list all its part sizes. Overline the ones that are non-overlined and remove the overline from the overlined parts. For example, starting with the overpartition $(8,6,6,4,4, \overline{4}, 2, \overline{1})$, we get $(\overline{8}, 6, \overline{6}, 4,4,4, \overline{2}, 1)$.

The main results of this work are asymptotic, following ideas that were used for partitions in $[5,9,10,19]$. Most of the properties we study will be asymptotic and will use analytic and probabilistic techniques, extensively developed for partitions, see for example $[1,12,14,23]$. Let us first recall a few results on partitions :

- The average number of distinct parts of a partition of $n$ is asymptotic to $\sqrt{6 n} / \pi$ as $n \rightarrow \infty[10]$.

- The average weight of the part sizes of a partition of $n$ is asymptotic to $6 n / \pi^{2}$ as $n \rightarrow \infty[10,19]$.

- The average number of parts of a partition of $n$ is asymptotic to $\sqrt{6 n} \ln n /(2 \pi)$ as $n \rightarrow \infty[16,18]$.

- The probability that a randomly chosen part size of a randomly chosen partition of $n$ has multiplicity $m$ approaches $1 /(m(m+1))$ as $n \rightarrow \infty[9]$.

- The expected multiplicity of a randomly chosen part size of a randomly chosen partition of $n$ is asymptotic to $(\ln n) / 2$ as $n \rightarrow \infty[9]$.

- The variance of the number of part sizes in a random partition is asymptotic to $\left(\frac{\sqrt{6}}{2 \pi}-\frac{\sqrt{54}}{\pi^{3}}\right) \sqrt{n}$, as $n \rightarrow \infty[13]$.

Following the remark of [11], we recall that at the origin of the Hardy-Ramanujan collaboration [14] started by "Ramanujan's false statement" that the coefficient of $q^{n}$ in $1 / \theta_{4}(q)$, which is $\bar{p}(n)$, is the nearest integer to

$$
\frac{1}{4 n}\left(\cosh \pi \sqrt{n}-\frac{\sinh \pi \sqrt{n}}{\pi \sqrt{n}}\right) .
$$

Then Hardy and Ramanujan proved that [15] :

(1) $\bar{p}(n)=\frac{1}{4 \pi} \frac{d}{d n} \frac{e^{\pi \sqrt{n}}}{\sqrt{n}}+\frac{\sqrt{3}}{2 \pi} \cos (2 n \pi / 3-1 / 6 \pi) \frac{d}{d n} \frac{e^{\pi \sqrt{n} / 3}}{\sqrt{n}}\left(1+O\left(n^{-1 / 4}\right)\right)$.

Using this asymptotic and some combinatorial and analytic arguments, we can get a collection of results proved in the following sections :

Theorem 1.1. The average number of part sizes of an overpartition of $n$ is :

$$
\frac{4 \ln 2}{\pi} \sqrt{n}
$$

as $n \rightarrow \infty$.

This trivially implies that the average number of overlined parts of an overpartition of $n$ is asymptotically : $\frac{2 \ln 2}{\pi} \sqrt{n}$. 
Theorem 1.2. The average number of parts of multiplicity $m$ or $m+1$ of an overpartition of $n$ is asymptotic to :

$$
\frac{4}{m(m+1) \pi} \sqrt{n}
$$

as $n \rightarrow \infty$.

Theorem 1.3. The average number of parts of an overpartition of $n$ is asymptotic to :

as $n \rightarrow \infty$.

$$
\sqrt{n} \frac{\ln n}{\pi}
$$

Theorem 1.4. The average weight of the overlined parts of an overpartition of $n$ is asymptotic to :

$$
\frac{n}{3}
$$

as $n \rightarrow \infty$.

Theorem 1.5. The probability that a randomly chosen part size of a randomly chosen overpartition of $n$ has multiplicity $m$ or $m+1$ approaches :

$$
\frac{1}{m(m+1) \ln 2} \text {. }
$$

as $n \rightarrow \infty$.

Theorem 1.6. The expected multiplicity of a part size of an overpartition of $n$ is asymptotic to :

$$
\text { as } n \rightarrow \infty \text {. } \frac{\ln n}{4 \ln 2}
$$

Finally we study the variance of the number of part sizes of a random overpartition of $n$ since it will be needed for the proofs of Theorems 1.5 and 1.6 :

Theorem 1.7. The variance of the number of part sizes in a random overpartition of $n$ is asymptotic to :

$$
\left(\frac{4(1-\ln 2)}{\pi}-\frac{\pi}{9}\right) \sqrt{n},
$$

as $n \rightarrow \infty$. Numerical value of the constant is about $.04163129 \ldots$.

We will start by a combinatorial part to know how to count the different statistics. Then we will go to asymptotics and finish with probabilities.

\section{Combinatorics}

Let $\bar{p}_{j}(n)$ be the number of overpartitions of $n$ in which at least one part is a $j$.

\section{Lemma 2.1}

$$
\bar{p}_{j}(n)=2 \sum_{i \geq 1}(-1)^{i-1} \bar{p}(n-i j)
$$

Proof. We prove this by induction. We show that $\bar{p}_{j}(n)=2 \bar{p}(n-j)-\bar{p}_{j}(n-$ $j$ ) if $n \geq j$ and 0 otherwise. The base case is obvious. Suppose that $n \geq j$. Overpartitions of $n$ that contain at least one part $j$ can be of two sorts : 
- $j$ appears at least once non-overlined. These are counted by $\bar{p}(n-j)$, as you can insert part $j$ to an overpartition of $n-j$ and create an overpartition of $n$ in which $j$ appears at least once non-overlined.

- $j$ appears once and is overlined. These are the overpartitions of $n-j$ where $j$ does not appear. These overpartitions are counted by $\bar{p}(n-j)-\bar{p}_{j}(n-j)$.

Let $\bar{p}_{j}^{(m)}(n)$ be the number of overpartitions of $n$ in which at least $m$ parts are equal to $j$.

\section{Lemma 2.2.}

$$
\bar{p}_{j}^{(m)}(n)=2 \sum_{i \geq 0}(-1)^{i} \bar{p}(n-(m+i) j)
$$

Proof. Same type of argument.

Let $\bar{p}_{i, j}(n)$ be the number of overpartitions of $n$ in which at least one part is an $i$ and at least one part is a $j$.

Lemma 2.3.

$$
\bar{p}_{i, j}(n)=4 \sum_{k, \ell \geq 1}(-1)^{k+\ell-2} \bar{p}(n-k i-\ell j)
$$

Proof. We prove this by induction. We show that $\bar{p}_{i, j}(n)=2 \bar{p}_{i}(n-j)-\bar{p}_{i, j}(n-j)$ if $n \geq i+j$ and 0 otherwise. The base case is obvious. Suppose that $n \geq i+j$. Overpartitions of $n$ that contain at least one part $i$ and one part $j$ can be of two sorts :

- $i$ appears and $j$ appears at least once non-overlined. These are counted by $\bar{p}_{i}(n-j)$.

- $i$ appears and $j$ appears once and is overlined. These are the overpartitions of $n-j$ where $i$ appears and $j$ does not appear. These overpartitions are counted by $\bar{p}_{i}(n-j)-\bar{p}_{i, j}(n-j)$.

Now let us apply these results. Let $I_{j}$ be the random variable which is 1 if at least one part is equal to $j$ in a random overpartition of $n$ and 0 otherwise. Let $\bar{D}_{n}$ be the random variable which expresses the number of part sizes of a random overpartition of $n$.

Lemma 2.4. The average number of part sizes in an overpartition of $n$ is :

$$
\mathbf{E} \bar{D}_{n}=\frac{2}{\bar{p}(n)} \sum_{i, j \geq 1}(-1)^{i-1} \bar{p}(n-i j) .
$$

Proof. From the definition of $\bar{D}_{n}$, we know that $\bar{D}_{n}=\sum_{j} I_{j}$. As $\mathbf{P}\left(I_{j}\right)=\frac{\bar{p}_{j}(n)}{\bar{p}(n)}$, $\mathbf{E} \bar{D}_{n}=\frac{1}{\bar{p}(n)} \sum_{j} \bar{p}_{j}(n)$. Using Lemma 2.1, we get the result.

Let $\bar{D}_{n, m}$ be the random variable which expresses the number of parts of multiplicity at least $m$ of a random overpartition of $n$.

Lemma 2.5. The average number of parts of multiplicity at least $m$ in an overpartition of $n$ is :

$$
\mathbf{E} \bar{D}_{n, m}=\frac{2}{\bar{p}(n)} \sum_{i, \ell \geq 1}(-1)^{i-1} \bar{p}(n-(m+i-1) \ell) .
$$


Proof. It is easy to see that $\mathbf{E} \bar{D}_{n, m}=\frac{1}{\bar{p}(n)} \sum_{\ell} \bar{p}_{\ell}^{(m)}(n)$. Using Lemma 2.2, we get the result.

Let $\bar{\Delta}_{n, m}$ be the random variable which expresses the number of parts of multiplicity $m$ or $m+1$ of a random overpartition of $n$.

Lemma 2.6. The average number of parts of multiplicity $m$ or $m+1$ in an overpartition of $n$ is :

$$
\mathbf{E} \bar{\Delta}_{n, m}=\frac{2}{\bar{p}(n)} \sum_{\ell \geq 1}(\bar{p}(n-m \ell)-\bar{p}(n-(m+1) \ell)) .
$$

Proof. It is easy to see that $\mathbf{E} \bar{\Delta}_{n, m}=\mathbf{E} \bar{D}_{n, m}-\mathbf{E} \bar{D}_{n, m+2}$

Let $\bar{K}_{n}$ be the random variable which expresses the number of parts of a random overpartition of $n$.

Lemma 2.7. The average number of parts in an overpartition of $n$ is :

$$
\mathbf{E} \bar{K}_{n}=\frac{2}{\bar{p}(n)} \sum_{i, j \geq 1} \bar{p}(n-(2 i-1) j) .
$$

Proof. It is easy to see that $\mathbf{E} \bar{K}_{n}=\frac{1}{\bar{p}(n)} \sum_{\ell, m} \bar{p}_{\ell}^{(m)}(n)$. Using Lemma 2.2, we compute and get the result.

Let $\bar{W}_{n}$ be the random variable which expresses the sum of the overlined parts of a random overpartition of $n$.

Lemma 2.8. The average value of the sum of overlined parts in an overpartition of $n$ is :

$$
\mathbf{E} \bar{W}_{n}=\frac{1}{\bar{p}(n)} \sum_{i, j \geq 1}(-1)^{i-1} j \bar{p}(n-i j) .
$$

Proof. It is easy to see that $\mathbf{E} \bar{W}_{n}=\frac{1}{\bar{p}(n)} \sum_{j} \frac{j \bar{p}_{j}(n)}{2}$. Using Lemma 2.1, we compute and get the result.

\section{3. АлумртотісS}

Most of the time we will need the first term from (1) :

$$
\bar{p}(n)=\frac{1}{8 n} \exp (\pi \sqrt{n})\left(1+O\left(n^{-1 / 2}\right)\right),
$$

as $n \rightarrow \infty$. This first term can also be obtained thanks to the general Meinardus theorem [1]. We will use that asymptotic formula to derive Theorems 1.1- 1.4. After some elementary manipulations the formula (2) yields

$$
\frac{\bar{p}(n-k i)}{\bar{p}(n)}= \begin{cases}\left(1+O\left(\frac{1}{n^{1 / 4}}\right)\right) e^{-\frac{\pi k i}{2 \sqrt{n}}} & \text { if } k i \leq n^{3 / 4} \\ O\left(e^{-\pi n^{1 / 4} / 2}\right) & \text { otherwise }\end{cases}
$$

We will use basic summation and integration techniques and omit some of the details of the calculations. No efforts were made to optimize the error terms. 
Proof of Theorem 1.1. From Lemma 2.4 and using (3) :

$$
\begin{aligned}
\mathbf{E} \bar{D}_{n} & =2 \sum_{i, k}(-1)^{i-1} \frac{\bar{p}(n-k i)}{\bar{p}(n)} \\
& =2 \sum_{k} \sum_{i \leq n^{3 / 4} / k}(-1)^{i-1} \exp \left(-\frac{\pi k i}{2 \sqrt{n}}\right)+O\left(n^{2} \exp \left(-\pi n^{1 / 4} / 2\right)\right) \\
& =2 \sum_{k} \frac{\exp \left(-\frac{\pi k}{2 \sqrt{n}}\right)}{1+\exp \left(-\frac{\pi k}{2 \sqrt{n}}\right)}+O(1) \\
& =2 \int_{1}^{\infty} \frac{\exp \left(-\frac{\pi x}{2 \sqrt{n}}\right)}{1+\exp \left(-\frac{\pi x}{2 \sqrt{n}}\right)} d x+O(1) \\
& =\frac{4 \sqrt{n}}{\pi} \ln (1+\exp (-\pi /(2 \sqrt{n})))+O(1) \\
& =\frac{4 \sqrt{n}}{\pi} \ln 2+O(1) .
\end{aligned}
$$

We get

$$
\mathbf{E} \bar{D}_{n} \sim \frac{4 \ln 2}{\pi} \sqrt{n}
$$

Proof of Theorem 1.2. From Lemma 2.6, we know that

$$
\mathbf{E} \bar{\Delta}_{n, m}=2 \sum_{i} \frac{\bar{p}(n-m i)-\bar{p}(n-(m+1) i)}{\bar{p}(n)}
$$

Using (3), we get

$$
\sum_{i} \frac{\bar{p}(n-m i)}{\bar{p}(n)}=\sum_{i \leq n^{3 / 4} / m} \frac{\bar{p}(n-m i)}{\bar{p}(n)}+O(1)=\frac{2}{m \pi} \sqrt{n}+O\left(n^{1 / 4}\right)
$$

Therefore,

$$
\mathbf{E} \bar{\Delta}_{n, m} \sim \frac{4}{m(m+1) \pi} \sqrt{n}
$$

Proof of Theorem 1.3. From Lemma 2.7, we know that

$$
\mathbf{E} \bar{K}_{n}=2 \sum_{i, j \geq 1} \frac{\bar{p}(n-(2 i-1) j)}{\bar{p}(n)}
$$


From (3),

$$
\begin{aligned}
\sum_{i, j} \frac{\bar{p}(n-(2 i-1) j)}{\bar{p}(n)} & =\sum_{(2 i-1) j \leq n^{3 / 4}} \frac{\bar{p}(n-(2 i-1) j)}{\bar{p}(n)}+O\left(n^{2} \exp \left(-\pi n^{1 / 4} / 2\right)\right) \\
& =\left(1+O\left(\frac{1}{n^{1 / 4}}\right)\right) \sum_{(2 i-1) j \leq n^{3 / 4}} \exp \left(-\frac{\pi(2 i-1) j}{2 \sqrt{n}}\right)+O(1) \\
& =\left(1+O\left(\frac{1}{n^{1 / 4}}\right)\right) \sum_{i \geq 1} \frac{\exp \left(-\frac{\pi(2 i-1)}{2 \sqrt{n}}\right)}{1-\exp \left(-\frac{\pi(2 i-1)}{2 \sqrt{n}}\right)}+O(1) \\
& =\int_{1}^{\infty} \frac{\exp \left(-\frac{\pi(2 x-1)}{2 \sqrt{n}}\right)}{1-\exp \left(-\frac{\pi(2 x-1)}{2 \sqrt{n}}\right)} d x+O\left(n^{1 / 2}\right) \\
& =\frac{\sqrt{n}}{\pi}\left(\frac{1}{2} \ln n+O(1)\right)
\end{aligned}
$$

Proof of Theorem 1.4. From Lemma 2.8, we know that

$$
\mathbf{E} \bar{W}_{n}=\sum_{i, j \geq 1}(-1)^{i-1} j \frac{\bar{p}(n-i j)}{\bar{p}(n)} .
$$

Using (3) and dropping the alternating sign in the error estimate we get

$$
\begin{aligned}
\mathbf{E} \bar{W}_{n} & =\sum_{i \cdot j \leq n^{3 / 4}}(-1)^{i-1} j \frac{\bar{p}(n-i j)}{\bar{p}(n)}+O(1) \\
& =\sum_{i \cdot j \leq n^{3 / 4}} j(-1)^{i-1} \exp \left(-\frac{\pi i j}{2 \sqrt{n}}\right)+O\left(\frac{1}{n^{1 / 4}}\right) \sum_{i \cdot j \leq n^{3 / 4}} j \exp \left(-\frac{\pi i j}{2 \sqrt{n}}\right) \\
& =\sum_{j} j \frac{\exp \left(-\frac{\pi j}{2 \sqrt{n}}\right)}{1+\exp \left(-\frac{\pi j}{2 \sqrt{n}}\right)}+O\left(\frac{1}{n^{1 / 4}}\right) \sum_{j} j \frac{\exp \left(-\frac{\pi j}{2 \sqrt{n}}\right)}{1-\exp \left(-\frac{\pi j}{2 \sqrt{n}}\right)} \\
& =\int_{1}^{\infty} \frac{x \exp \left(-\frac{\pi x}{2 \sqrt{n}}\right)}{1+\exp \left(-\frac{\pi x}{2 \sqrt{n}}\right)} d x+O\left(n^{3 / 4}\right) \\
& =\frac{4 n}{\pi^{2}} \int_{0}^{\infty} \frac{x \exp (-x)}{1+\exp (-x)} d x+O\left(n^{3 / 4}\right) \\
& =\frac{n}{3}+O\left(n^{3 / 4}\right) .
\end{aligned}
$$

\section{Probabilistic argument}

To prove Theorems 1.5 and 1.6 we will need a concentration result for $\bar{D}_{n}$ :

$$
\operatorname{var} \bar{D}_{n}=O(\sqrt{n})
$$

which is, of course, a special case of Theorem 1.7.

Proof of Theorem 1.5. The probability in question is $\mathbf{E}\left(\bar{\Delta}_{n, m} / \bar{D}_{n}\right)$. Set $c=\frac{4 \ln 2}{\pi}$. Then, from Theorem $1.1 \mathbf{E} \bar{D}_{n} \sim c \sqrt{n}$. Using that, (4), and Chebyshev's inequality 
with $t_{n} \sim n^{3 / 8}$, we infer that

$$
\mathbf{P}\left(\left|\bar{D}_{n}-c \sqrt{n}\right| \geq n^{3 / 8}\right) \leq \frac{\operatorname{var} \bar{D}_{n}}{n^{3 / 4}}=O\left(n^{-1 / 4}\right) .
$$

With that given, using $\bar{\Delta}_{n, m} / \bar{D}_{n} \leq 1$ and $\bar{\Delta}_{n, m}=O(\sqrt{n})$, we may write:

$$
\begin{aligned}
\mathbf{E} \frac{\bar{\Delta}_{n, m}}{\bar{D}_{n}} & =\mathbf{E} \frac{\bar{\Delta}_{n, m}}{\bar{D}_{n}} I_{\left|\bar{D}_{n}-c \sqrt{n}\right|<t_{n}}+\mathbf{E} \frac{\bar{\Delta}_{n, m}}{\bar{D}_{n}} I_{\left|\bar{D}_{n}-c \sqrt{n}\right| \geq t_{n}} \\
& \sim \mathbf{E} \frac{\bar{\Delta}_{n, m}}{c \sqrt{n} \pm t_{n}} I_{\left|\bar{D}_{n}-c \sqrt{n}\right|<t_{n}}+O\left(\mathbf{P}\left(\left|\bar{D}_{n}-c \sqrt{n}\right| \geq t_{n}\right)\right) \\
& =\mathbf{E} \frac{\bar{\Delta}_{n, m}}{c \sqrt{n} \pm t_{n}}-\mathbf{E} \frac{\bar{\Delta}_{n, m}}{c \sqrt{n} \pm t_{n}} I_{\left|\bar{D}_{n}-c \sqrt{n}\right| \geq t_{n}}+O\left(n^{-1 / 4}\right) \\
& =\frac{\mathbf{E} \bar{\Delta}_{n, m}}{\mathbf{E} \bar{D}_{n} \pm o\left(\mathbf{E} \bar{D}_{n, m}\right)}+o(1) .
\end{aligned}
$$

Now apply Theorems 1.1 and 1.2.

Proof of Theorem 1.6. The average multiplicity is $\mathbf{E}\left(\bar{K}_{n} / \bar{D}_{n}\right)$. We would like to follow the same argument as above. One difference, however, is that the quantity $\bar{\Delta}_{m, n} / \bar{D}_{n}$ in the previous proof was bounded by 1 , whereas $\bar{K}_{n} / \bar{D}_{n}$ is not. Thus, more care is needed. Nonetheless, as before we write:

$$
\begin{aligned}
\mathbf{E} \frac{\bar{K}_{n}}{\bar{D}_{n}} & =\mathbf{E} \frac{\bar{K}_{n}}{\bar{D}_{n}} I_{\left|\bar{D}_{n}-c \sqrt{n}\right|<t_{n}}+\mathbf{E} \frac{\bar{K}_{n}}{\bar{D}_{n}} I_{\left|\bar{D}_{n}-c \sqrt{n}\right| \geq t_{n}} \\
& \sim \mathbf{E} \frac{\bar{K}_{n}}{c \sqrt{n} \pm t_{n}} I_{\left|\bar{D}_{n}-c \sqrt{n}\right|<t_{n}}+\mathbf{E} \frac{\bar{K}_{n}}{\bar{D}_{n}} I_{\left|\bar{D}_{n}-c \sqrt{n}\right| \geq t_{n}} \\
& =\frac{\mathbf{E} \bar{K}_{n}}{c \sqrt{n} \pm t_{n}}-\mathbf{E} \frac{\bar{K}_{n}}{c \sqrt{n} \pm t_{n}} I_{\left|\bar{D}_{n}-c \sqrt{n}\right| \geq t_{n}}+\mathbf{E} \frac{\bar{K}_{n}}{\bar{D}_{n}} I_{\left|\bar{D}_{n}-c \sqrt{n}\right| \geq t_{n}} .
\end{aligned}
$$

By Theorems 1.1 and 1.3, as long as $t_{n}=o(\sqrt{n})$ the first term is asymptotic to $\frac{\ln n}{4 \ln 2}$ so it suffices to show that each of the remaining two is $o(\log n)$. Set $t_{n}=$ $O\left(\sqrt{n} / \log ^{1 / 4} n\right)$. Since $\bar{K}_{n} \leq n$, using Chebyshev's inequality and (4) we see that the first of the two is bounded in absolute value by

$$
O(\sqrt{n}) \frac{\operatorname{var} \bar{D}_{n}}{t_{n}^{2}}=O\left(\log ^{1 / 2} n\right) .
$$

The toughest task is to bound the last term. First, $\left|\bar{D}_{n}-c \sqrt{n}\right| \geq t_{n}$ means that either $\bar{D}_{n} \geq c \sqrt{n}+t_{n}$ or $\bar{D}_{n} \leq c \sqrt{n}-t_{n}$, and in the first case

$$
\mathbf{E} \frac{\bar{K}_{n}}{\bar{D}_{n}} I_{\bar{D}_{n} \geq c \sqrt{n}+t_{n}}=O(\sqrt{n}) \mathbf{P}\left(\bar{D}_{n} \geq c \sqrt{n}+t_{n}\right)=O\left(\frac{n}{t_{n}^{2}}\right)=O\left(\log ^{1 / 2} n\right) .
$$

It remains to bound

$$
\mathbf{E} \frac{\bar{K}_{n}}{\bar{D}_{n}} I_{\bar{D}_{n} \leq c \sqrt{n}-t_{n}}
$$

Let $0<\varepsilon<c=(4 \ln 2) / \pi$ and split the set $\left\{\bar{D}_{n} \leq c \sqrt{n}-t_{n}\right\}$ in two pieces $\left\{\bar{D}_{n} \leq \varepsilon \sqrt{n}\right\}$ and $\left\{\varepsilon \sqrt{n}<\bar{D}_{n} \leq c \sqrt{n}-t_{n}\right\}$. On the latter set $\bar{K}_{n} / \bar{D}_{n} \leq \sqrt{n} / \varepsilon$ so that the expectation over that set is bounded by

$$
\begin{gathered}
\frac{\sqrt{n}}{\varepsilon} \mathbf{P}\left(\varepsilon \sqrt{n}<\bar{D}_{n} \leq c \sqrt{n}-t_{n}\right) \leq \frac{\sqrt{n}}{\varepsilon} \mathbf{P}\left(\bar{D}_{n}-c \sqrt{n} \leq-t_{n}\right) \\
=O(\sqrt{n}) \mathbf{P}\left(\left|\bar{D}_{n}-c \sqrt{n}\right| \geq t_{n}\right)=O\left(\log ^{1 / 2} n\right),
\end{gathered}
$$


by exactly the same argument as before. Finally, since $\bar{K}_{n} \leq n$,

$$
\mathbf{E} \frac{\bar{K}_{n}}{\bar{D}_{n}} I_{\bar{D}_{n} \leq \varepsilon \sqrt{n}} \leq n \mathbf{P}\left(\bar{D}_{n} \leq \varepsilon \sqrt{n}\right),
$$

and the proof will be complete once we prove

Lemma 4.1. There exist $0<\varepsilon<c, \eta>0$ such that as $n \rightarrow \infty$

$$
\mathbf{P}\left(\bar{D}_{n} \leq \varepsilon \sqrt{n}\right) \leq e^{-\eta \sqrt{n}}
$$

Proof. Let $u$ be any positive number. Then

$$
\mathbf{P}\left(\bar{D}_{n} \leq \varepsilon \sqrt{n}\right)=\mathbf{P}\left(e^{-u \bar{D}_{n}} \geq e^{-u \varepsilon \sqrt{n}}\right) \leq e^{u \varepsilon \sqrt{n}} \mathbf{E} e^{-u \bar{D}_{n}},
$$

and we need to bound the last expectation. To this end we use a relationship between overpartitions and classical partitions, namely that every partition with $k$ part sizes gives $2^{k}$ different overpartitions (obtained by overlining or not each of the $k$ part sizes). Thus, the uniform probability measure on the set of all overpartitions of $n$ corresponds to the uniform probability measure $\mathbf{Q}$ on the set $\Omega_{n}$ of all partitions, weighted by a factor $2^{D_{n}}$, where $D_{n}$ is the number of distinct parts in a partition of $n$. (In other word, $\bar{D}_{n}$ and $D_{n}$ represent the same quantity on $\Omega_{n}$ but considered with respect to two different probability measures on $\Omega_{n}$.) Thus, letting $\mathbf{E}_{\mathbf{Q}}$ denote the expectation with respect to $\mathbf{Q}$ on $\Omega_{n}$, we have

$$
\begin{aligned}
\mathbf{E} e^{-u \bar{D}_{n}} & =\sum_{k} \mathbf{E}\left(e^{-u \bar{D}_{n}} \mid \bar{D}_{n}=k\right) \mathbf{P}\left(\bar{D}_{n}=k\right)=\sum_{k} e^{-u k} \frac{2^{k} \mathbf{Q}\left(D_{n}=k\right)}{\sum_{j \geq 1} 2^{j} \mathbf{Q}\left(D_{n}=j\right)} \\
& =\frac{\mathbf{E}_{\mathbf{Q}} 2^{D_{n}} e^{-u D_{n}}}{\mathbf{E}_{\mathbf{Q}} 2^{D_{n}}} \leq O(1) \cdot 2^{-\frac{\sqrt{6}}{\pi} \sqrt{n}} \mathbf{E}_{\mathbf{Q}} 2^{D_{n}} e^{-u D_{n}},
\end{aligned}
$$

where in the last step we used $\mathbf{E}_{\mathbf{Q}} D_{n}=\frac{\sqrt{6}}{\pi} \sqrt{n}+O(1)$, and convexity of $x \rightarrow 2^{x}$. In order to bound the numerator we resort to a conditioning trick introduced by Fristedt [12] (see e.g. [5, 9] for applications along the lines we will use below). Namely, let $X_{j}$ be a geometric random variable with parameter $1-q^{j}, 0<q<1$, (that is $\mathbf{P}\left(X_{j}=i\right)=q^{j i}\left(1-q^{j}\right)$ for $\left.i=0,1, \ldots\right)$ and suppose that $X_{j}, j=1,2 \ldots$, are independent. If $\lambda$ is chosen uniformly at random (i.e. according to the measure Q) then the joint distribution of multiplicities of part sizes $\left(m_{1}, m_{2}, \ldots\right)$ is equal to that of $\left(X_{1}, X_{2}, \ldots\right)$ conditioned on $\sum_{j} j X_{j}=n$. Since $D_{n}=\sum_{j} I_{m_{j} \geq 1}$, letting $I_{j}=I_{X_{j} \geq 1}$ we get

$$
\begin{aligned}
\mathbf{E}_{\mathbf{Q}} 2^{D_{n}} e^{-u D_{n}} & =\mathbf{E}_{\mathbf{Q}} e^{(\ln 2-u) \sum_{j} I_{m_{j} \geq 1}}=\mathbf{E}\left(e^{(\ln 2-u) \sum I_{j}} \mid \sum_{j} j X_{j}=n\right) \\
& \leq \frac{\mathbf{E} e^{(\ln 2-u) \sum I_{j}}}{\mathbf{P}\left(\sum_{j} j X_{j}=n\right)}
\end{aligned}
$$

As was noted by Fristedt, the choice $q=\exp \left(-\frac{\pi}{\sqrt{6 n}}\right)$ makes denominator of order $n^{-3 / 4}$. Further, since $I_{j}$ 's are independent and $\mathbf{E} e^{s I_{j}}=1+\left(e^{s}-1\right) q^{j} \leq \exp \left(\left(e^{s}-\right.\right.$ 1) $\left.q^{j}\right)$,

$$
\begin{aligned}
\mathbf{E} e^{(\ln 2-u) \sum I_{j}} & =\prod_{j} \mathbf{E} e^{(\ln 2-u) I_{j}} \leq e^{\left(2 e^{u}-1\right) \sum_{j \geq 1} q^{j}}=e^{\left(2 e^{-u}-1\right) \frac{q}{1-q}} \\
& =O(1) \cdot e^{\frac{\sqrt{6}}{\pi}\left(2 e^{-u}-1\right)} .
\end{aligned}
$$


Combining all of this with (5) and (6) we get

$$
\mathbf{P}\left(\bar{D}_{n} \leq \varepsilon \sqrt{n}\right) \leq O\left(n^{3 / 4}\right) \cdot \exp \left(\sqrt{n}\left\{u \varepsilon+\frac{\sqrt{6}}{\pi}\left(2 e^{-u}-1-\ln 2\right)\right\}\right)
$$

and it is clear that the lemma will be proved if there is a choice of $0<\varepsilon<c$ and $u>0$ for which

$$
f_{\varepsilon}(u)=u \varepsilon+\frac{\sqrt{6}}{\pi}\left(2 e^{-u}-1-\ln 2\right) \leq-\eta_{0}<0 .
$$

(Of course, the factor $O\left(n^{3 / 4}\right)$ is inessential since its influence can be nullified by choosing $0<\eta<\eta_{0}$.) For that it is enough to check that $\inf _{u} f_{\varepsilon}(u)<0$. Now,

$$
f_{\varepsilon}^{\prime}(u)=\varepsilon-2 \frac{\sqrt{6}}{\pi} e^{-u}
$$

which is 0 if $u=u_{0}=\ln \left(\frac{2 \sqrt{6}}{\varepsilon \pi}\right)$ (and we need $\varepsilon<2 \sqrt{6} / \pi$ to ensure that $u_{0}>0$ ). It is now clear that we can make $f_{\varepsilon}\left(u_{0}\right)$ negative by looking at the graph of a function

$$
h(\varepsilon)=f_{\varepsilon}\left(u_{0}\right)=\varepsilon \ln \left(\frac{2 \sqrt{6}}{\varepsilon \pi}\right)+\frac{\sqrt{6}}{\pi}\left(\frac{\varepsilon \pi}{\sqrt{6}}-1-\ln 2\right)
$$

near zero. Specifically, we may choose $\varepsilon_{0}=\sqrt{6} /(2 \pi)<c=(4 \ln 2) / \pi$ which gives $\eta_{0}:=h\left(\varepsilon_{0}\right)=-\sqrt{6} /(2 \pi)$.

\section{THE VARIANCE}

Proof of Theorem 1.7. The proof is along the arguments given in the previous section. We will need more precision however to take the full advantage of sign alternations. Using a full strength of (1) we see that

$$
\bar{p}(n) \sim \frac{e^{\pi \sqrt{n}}}{8 n}\left(1-\frac{1}{\pi \sqrt{n}}+O\left(e^{-2 \pi \sqrt{n} / 3}\right)\right)
$$

Neglecting the exponentially small error gives

$$
\begin{aligned}
\frac{\bar{p}(n-m)}{\bar{p}(n)} & \sim \frac{e^{\pi(\sqrt{n-m}-\sqrt{n})}\left(1-\pi^{-1} / \sqrt{n-m}\right)}{(1-m / n)\left(1-\pi^{-1} / \sqrt{n}\right)} \\
& =\frac{e^{\pi \sqrt{n}(\sqrt{1-m / n}-1)}}{(1-m / n)\left(1-\pi^{-1} / \sqrt{n}\right)}\left(1-\frac{1}{\pi \sqrt{n} \sqrt{1-m / n}}\right)
\end{aligned}
$$


We have

$$
\begin{aligned}
\operatorname{var} \bar{D}_{n} & =\mathbf{E} \bar{D}_{n}^{2}-\left(\mathbf{E} \bar{D}_{n}\right)^{2}=\mathbf{E}\left(\sum_{k} I_{k}\right)^{2}-\left(\frac{1}{\bar{p}(n)} \sum_{\ell} \bar{p}_{\ell}(n)\right)^{2} \\
& =\mathbf{E} \bar{D}_{n}+\frac{1}{\bar{p}(n)} \sum_{k \neq \ell} \bar{p}_{\ell, k}(n)-\frac{1}{\bar{p}^{2}(n)} \sum_{k, \ell} \bar{p}_{\ell}(n) \bar{p}_{k}(n) \\
& =\mathbf{E} \bar{D}_{n}+4 \sum_{i, j, k, \ell \geq 1}(-1)^{i+j-2}\left\{\frac{\bar{p}(n-k i-\ell j)}{\bar{p}(n)}-\frac{\bar{p}(n-j \ell)}{\bar{p}(n)} \cdot \frac{\bar{p}(n-k i)}{\bar{p}(n)}\right\} \\
(8) \quad & :=\mathbf{E} \sum_{i, j, k}(-1)^{i+j-2} \frac{\bar{p}(n-k(i+j))}{\bar{p}(n)}
\end{aligned}
$$

We will now use $(7)$ and expansions of $1 /(1-x)$ and $1 / \sqrt{1-x}$ into their power series. Our first step will be to discard all terms whose contribution (even after ignoring sign alternation) is negligible. We begin with the triple sum $T$. Using (7) and the expansions with $x=k(i+j) / n$ we see that the summand in $T$ is

$$
(-1)^{i+j-2} e^{\pi \sqrt{n}(\sqrt{1-k(i+j) / n}-1)}\left\{1+\frac{k(i+j)}{n}-\frac{1}{2 \pi} \frac{k(i+j)}{n^{3 / 2}}+O\left(\frac{k^{2}(i+j)^{2}}{n^{2}}\right)\right\}
$$

and we claim that the contribution from the sum of the absolute values of the last two terms is $o(\sqrt{n})$. Indeed, we have

$$
\begin{aligned}
\sum_{i, j, k} k(i+j) e^{\pi \sqrt{n}(\sqrt{1-k(i+j) / n}-1)} & \sim 2 \sum_{k} k \sum_{j} e^{-k j \pi /(2 \sqrt{n})} \sum_{i} e^{-i k \pi /(2 \sqrt{n})} \\
& =2 \sum_{k} \frac{k \exp (-k \pi / \sqrt{n})}{(1-\exp (-k \pi /(2 \sqrt{n})))^{3}} \\
& =O\left(n^{3 / 2}\right) .
\end{aligned}
$$

If we observe as in the proof of Theorem 1.1 that we may restrict the indices $i, j, k$ to satisfy, say, $k(i+j)=O\left(n^{5 / 6}\right)$, (for all the terms corresponding to $k(i+j) \geq 2 n^{5 / 6}$ are bounded by $e^{-\pi n^{1 / 3}}$ and there are polynomially many of them) we conclude that the contribution from the last two terms is

$$
O(1)+O\left(\frac{k(i+j)}{n^{2}}\right) \sum_{i, j, k} k(i+j) e^{-\pi k(i+j) / 2(\sqrt{n})}=O\left(n^{1 / 3}\right) .
$$

Consequently,

$$
\begin{aligned}
T & \sim \sum_{k, i, j}(-1)^{i+j-2}\left(1+\frac{k(i+j)}{n}\right) e^{-k(i+j) \pi /(2 \sqrt{n})} \\
& =\sum_{k \geq 1}\left\{\frac{e^{-k \pi / \sqrt{n}}}{\left(1+e^{-k \pi /(2 \sqrt{n})}\right)^{2}}+2 \frac{k}{n} \frac{e^{-k \pi / \sqrt{n}}}{\left(1+e^{-k \pi /(2 \sqrt{n}))^{3}}\right.}\right\} .
\end{aligned}
$$

By calculations similar to those in the proofs of Theorems 1.1-1.4 we see that the first term is asymptotic to $2 \sqrt{n}(\ln 2-1 / 2) / \pi$ and the second is $O(1)$. Hence

$$
4 T \sim \frac{8 \ln 2-4}{\pi} \sqrt{n}
$$


Contribution of the quadruple sum $Q$ is computed along the same lines; we use

$$
\sum_{k, i, \ell, j} \frac{k i+\ell j}{n^{3 / 2}} \exp \left(\pi \sqrt{n}\left(\sqrt{1-\frac{k i+\ell j}{n}}-1\right)\right)=O\left(\log ^{2} n\right)
$$

and

$$
\sum_{k, i, \ell, j} \frac{(k i+\ell j)^{2}}{n^{2}} \exp \left(\pi \sqrt{n}\left(\sqrt{1-\frac{k i+\ell j}{n}}-1\right)\right)=O\left(\log ^{2} n\right)
$$

to see that the term $\bar{p}(n-k i-\ell j) / \bar{p}(n)$ is asymptotic to

$$
\left(1+\frac{k i+\ell j}{n}\right) \exp \left(\pi \sqrt{n}\left(\sqrt{1-\frac{k i+\ell j}{n}}-1\right)\right)
$$

Similarly,

$$
\frac{\bar{p}(n-k i)}{\bar{p}(n)} \frac{\bar{p}(n-\ell j)}{\bar{p}(n)} \sim\left(1+\frac{k i+\ell j}{n}\right) \exp \left(\pi \sqrt{n}\left(\sqrt{1-\frac{k i}{n}}-1+\sqrt{1-\frac{\ell j}{n}}-1\right)\right) .
$$

If we now use

$$
\begin{aligned}
& \exp \left(\pi \sqrt{n}\left(\sqrt{1-\frac{m}{n}}-1\right)\right)=\exp \left(-\frac{\pi m}{2 \sqrt{n}}+\pi \sqrt{n}\left(\sqrt{1-\frac{m}{n}}-1+\frac{m}{2 n}\right)\right) \\
& =\exp \left(-\frac{\pi m}{2 \sqrt{n}}\right)\left(1-\pi \frac{m^{2}}{8 n^{3 / 2}}+O\left(\frac{m^{3}}{n^{5 / 2}}\right)\right)
\end{aligned}
$$

and apply it with $m=k i+\ell j, k i$, and $\ell j$ we get that the significant term in the difference in the expression for $Q$ is

$$
\begin{gathered}
\left(1+\frac{k i+\ell j}{n}\right) e^{-\pi \frac{k i+\ell j}{2 \sqrt{n}}}\left(1-\frac{\pi}{8 n^{3 / 2}}(k i+\ell j)^{2}-1+\frac{\pi}{8 n^{3 / 2}}\left(k^{2} i^{2}+\ell^{2} j^{2}\right)\right) \\
=-\pi\left(1+\frac{k i+\ell j}{n}\right) \frac{k i \ell j}{4 n^{3 / 2}} e^{-\pi \frac{k i+\ell j}{2 \sqrt{n}}} \sim-\pi \frac{k i \ell j}{4 n^{3 / 2}} e^{-\pi \frac{k i+\ell j}{2 \sqrt{n}}}
\end{gathered}
$$

where the last approximation is justified by

$$
\begin{aligned}
\frac{1}{n^{5 / 2}} \sum_{k, i, \ell, j} k^{2} i^{2} \ell j e^{-\pi \frac{k i+\ell j}{2 \sqrt{n}}} & =\frac{1}{n^{5 / 2}} \sum_{k, i} k^{2} i^{2} e^{-\pi \frac{k i}{2 \sqrt{n}}} \sum_{\ell, j} \ell j e^{-\pi \frac{\ell j}{2 \sqrt{n}}} \\
& =\frac{1}{n^{5 / 2}} O\left(n^{3 / 2} \log n\right) O(n \log n)=O\left(\log ^{2} n\right)
\end{aligned}
$$

Consequently,

$$
\begin{aligned}
4 Q & \sim-\frac{\pi}{n^{3 / 2}} \sum_{i, k, \ell, j}(-1)^{i+j-2} k i \ell j e^{-\pi \frac{k i+\ell j}{2 \sqrt{n}}}=-\frac{\pi}{n^{3 / 2}}\left(\sum_{i, k}(-1)^{i-1} k i e^{-\pi \frac{k i}{2 \sqrt{n}}}\right)^{2} \\
& =-\frac{\pi}{n^{3 / 2}}\left(\sum_{k} k \frac{e^{-\pi \frac{k}{2 \sqrt{n}}}}{1+e^{-\pi \frac{k}{2 \sqrt{n}}}}\right)^{2} \sim-\frac{\pi}{n^{3 / 2}}\left(\frac{n}{3}\right)^{2}=-\frac{\pi}{9} \sqrt{n} .
\end{aligned}
$$

Combining this with (9) and Theorem 1.1 completes the proof. 


\section{Conclusion}

The purpose of this paper was to study the asymptotics of the number of parts in overpartitions. The behavior of these parameters looks a lot like partitions but the proofs of the results do not follow directly for results known on partitions. Overpartitions are partitions counted with the weight $2^{k}$ where $k$ is the number of part sizes. This weight gives the following analogues :

- The probability that a randomly chosen part size of a randomly chosen partition (resp. overpartition) of $n$ has multiplicity $m$ (resp. $m$ or $m+1$ ) approaches $1 /(m(m+1))$ (resp. $1 /(m(m+1) \ln 2))$ as $n \rightarrow \infty$.

- The expected multiplicity of a randomly chosen part size of a randomly chosen partition (resp. overpartition) of $n$ approaches $\ln n / 2(\operatorname{resp} .(\ln n /(4 \ln 2))$ as $n \rightarrow \infty$.

\section{REFERENCES}

[1] G.E. Andrews, The theory of partitions, Cambridge Univ. Press (1984).

[2] L. Bégin, J.F. Fortin, P. Jacob and P. Mathieu, Fermionic characters for graded parafermions, Nuclear Phys. B 659 (2003) 365-386.

[3] C. Bessenrodt and I. Pak, Partition congruences by involutions, Europ. J. Combinatorics, to appear.

[4] S. Corteel, Particle seas and basic hypergeometric series, Adv. in Appl. Math., 31 (2003) no 1, 199-214.

[5] E.R. Canfield, S. Corteel and P. Hitczenko, Partitions with rth difference non-negative, Adv. in Appl. Math., 27 (2001), 298-317.

[6] S. Corteel and J. Lovejoy, Frobenius partitions and the combinatorics of Ramanujan's ${ }_{1} \psi_{1}$ summation, J. Combin. Theory Ser. A, 97 (2002) 179-183.

[7] S. Corteel and J. Lovejoy, Overpartitions, Trans. Amer. Math. Soc., 356 (2004), 1623-1635.

[8] S. Corteel, J. Lovejoy and A.J. Yee, Overpartitions and generating functions for generalized Frobenius partitions, Trends in Mathematics (Birkhauser), to appear.

[9] S. Corteel, B. Pittel, C.D. Savage and H.S. Wilf, On the multiplicity of parts in a random partition, Random Struct. Algorithms, 14 (1999), 185-197.

[10] P. Erdös and J. Lehner, The distribution of the number of summands in the partitions of a positive integer, Duke Math. J. 8 (1941) 335-345.

[11] J.F. Fortin, P. Jacob and P. Mathieu, Jagged partitions, preprint. arXiv:math.CO/0310079.

[12] B. Fristedt, The structure of random partitions of large integers, Trans. Amer. Math. Soc. 337 (1993), 703-735.

[13] W.M.Y. Goh and E. Schmutz, The number of distinct part sizes in a random integer partition, J. Combin. Theory Ser. A, 69 (1995) 149-158.

[14] G.H. Hardy, Ramanujan, Cambridge Univ. Press (1940).

[15] G.H. Hardy and Ramanujan, Asymptotic formulae in combinatory analysis, Proc. London Math. Soc. 2, XVII (1918) 75-115.

[16] K. Husimi, Partition numerorum as occurring in a problem of nuclear physics, Proceedings of the Physico-Mathematical Society of Japan, 20 (1938), 912-925.

[17] J.T. Joichi and D. Stanton, Bijective proofs of basic hypergeometric series identities. Pacific J. Math. 127 (1987), no. 1, 103-120.

[18] I. Kessler and M. Livingston, The expected number of parts in a partition of $n$, Monatsh. Math., 81 (1976) 203-212.

[19] A. Knopfmacher and M.E. Mays, The sum of distinct parts in compositions and partitions, Bull. Inst. Combin. Appl., 25 (1999), 66-78.

[20] J. Lovejoy, Gordon's theorem for overpartitions, J. of Combin. Theory Ser. A, 103 (2003) 393-401.

[21] J. Lovejoy, Overpartitions and real quadratic fields, J. Number Theory, 106 (2004) 178-186.

[22] J. Lovejoy, Overpartition theorems of the Rogers-Ramanujan type, J. London Math Soc., (2) 69 (2004) 562-574.

[23] H. Rademacher, Topics in analytic number theory, Springer Verlag (1973). 
[24] A.J. Yee, Combinatorial proofs of Ramanujan's ${ }_{1} \psi_{1}$ summation and the q-Gauss summation, J. Combin. Theory Series A, 105 (2004) 63-77.

CNRS PRiSM, UVSQ, Versailles, France

E-mail address: syl@prism.uvsq.fr

Drexel University, Philadelphia, USA

E-mail address: phitczenko@mcs.drexel.edu 